\title{
Influence of varying magnetic order in external magnetic field on the electronic structure and Fermi surface within the t-J model
}

\author{
V.I. Kuz'min ${ }^{+1)}$, S. V. Nikolaev ${ }^{+*}$, S. G. Ovchinnikov ${ }^{+* \nabla}$ \\ ${ }^{+}$L.V. Kirensky Institute of Physics SB RAS, 660036 Krasnoyarsk, Russia \\ * Siberian Federal University, 660041 Krasnoyarsk, Russia

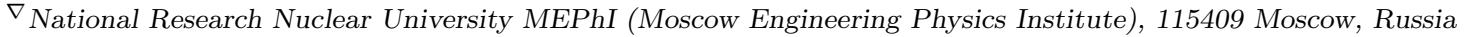

Submitted 11 November 2015 December 2015*

\begin{abstract}
We investigate the electronic structure of the two-dimensional $t$ - $J$ model in a transverse external static magnetic field with canted long-range magnetic order using cluster perturbation theory. Distribution of spectral weight in the whole range of fields from zero to ferromagnetic saturation is explored. We demonstrate the possibility of a sharp change in a distribution of spectral weight in the Brillouin zone at the Fermi level associated with the magnetic correlations when varying magnetic field.
\end{abstract}

\section{INTRODUCTION}

The electronic structure and Fermi surface (FS) of two-dimensional (2D) strongly correlated electron systems are sensitive to various reconstructions induced by symmetry breaking, complicating thus even more the puzzle of electronic properties of such compounds. Considerable attention is drawn to this problem by the investigations of quantum oscillations in high-temperature superconductors (HTSC).

Quantum oscillations in HTSC were first observed in the hole-underdoped yttrium compounds [1-4], then in the hole-overdoped $\mathrm{Tl}_{2} \mathrm{Ba}_{2} \mathrm{CuO}_{6+\delta}$ [5-7], electronunderdoped $\mathrm{Nd}_{2-\mathrm{x}} \mathrm{Ce}_{\mathrm{x}} \mathrm{CuO}_{4}$ [8] and hole-underdoped $\mathrm{HgBa}_{2} \mathrm{CuO}_{4+\delta}$ [9]. The summary data of these experiments show that oscillation frequencies in the underdoped and overdoped compounds differ by an order of magnitude, this way revealing the drastic transformation of the FS with doping. A similar result was recently obtained within the strong coupling approach to the Hubbard model [10]. In general, a comparable conclusion follows from the experiments on angle-resolved photoemission spectroscopy (ARPES) [11]. However, as for hole-underdoped cuprates, on the one hand, there are Fermi arcs of ARPES $[12,13]$ which are consistent with the calculations within the Hubbard and $t$ - $J$ models resulting in a hole pocket in the nodal direction [14-19]. On the other hand, it was shown that Hall and Seebeck coefficients become negative in high magnetic fields $[2,20]$, indicating at the existence of electron pocket(s). Resent data [21] on quantum oscillations in $\mathrm{YBa}_{2} \mathrm{Cu}_{3} \mathrm{O}_{\mathrm{y}}$ for hole doping $p=0.108$ agrees with the FS consisting of a nodal electron pocket (which was first proposed in [22]) accompanied by two small hole

\footnotetext{
1) e-mail: kuz@iph.krasn.ru
}

pockets as it was obtained from the calculations [23] within the charge-density wave (CDW) phase. Longrange CDW was in turn reported to emerge in a magnetic field [24] in $\mathrm{La}_{2-\mathrm{x}} \mathrm{Ba}_{\mathrm{x}} \mathrm{CuO}_{4}$ and $\mathrm{YBa}_{2} \mathrm{Cu}_{3} \mathrm{O}_{\mathrm{y}}$ in the vicinity of $p=1 / 8$.

Therefore, it is interesting to study the magnetic field-driven evolution of the FS of 2D strongly correlated systems at fixed doping. Although the energy of reasonably strong magnetic fields is rather small compared to the scale of the electronic structure, it may be important that strictly speaking, the experiments on quantum oscillations are accompanied by the change of symmetry with a net magnetic moment due to an applied magnetic field, while in the absence of external field there is short-range antiferromagnetic (AFM) order without total magnetization or long-range AFM. Moreover, the charge ordering in the vicinity of hole concentration $p=1 / 8$ complicated the FS even more. Due to the intrinsic interrelation of electron hopping and underlying magnetic or charge order in strongly correlated materials the field-induced change of magnetic symmetry may result in a strong effect on the electronic structure and Fermi surface.

In this paper, inspired by the experiments on quantum oscillations, we study the evolution of the FS in the whole range of magnetic fields from zero to saturation field, at which ferromagnetic alignment of spins is achieved, although for undoped cuprates such fields $h \sim J$ (where $J$ is the interatomic exchange interaction between neighboring spins, $J \sim 0.1 \mathrm{eV}$ ) are far out of reach of the present experimental abilities. The situation in hole-doped cuprates becomes extremely complex at doping levels $p \gtrsim 0.05$ due to the presence of incommensurate magnetic [25] and charge-density wave or- 
ders. We do not account for such density wave phases and investigate only the case of lower dopings relating to long ranged AFM order or short-range order with significant correlation length, both relevant for hole-doped cuprates [26]. Particularly, we examine whether a constant transverse magnetic field may cause a noticeable effect on the FS of hole-doped cuprate superconductors in experimentally achievable fields by means of the underlying magnetic order, focusing on the AFM underdoped case at zero temperature in the absence of the field. We apply a slightly modified version of the cluster perturbation theory (CPT) [27] to take into account long-range canted magnetic order and short-range nearest neighbor correlations simultaneously and study the $2 \mathrm{D} t$ - $J$ model $[28,29]$. We obtain a radical field-induced evolution of the FS in the whole range of fields from zero to saturation field ( $8 S J$ in the Heisenberg model [30], where $S$ is the value of on-site spin).

\section{BRIEF OVERVIEW OF THE METHOD}

Let us consider the $t-J$ model on a $2 \mathrm{D}$ square lattice in an applied constant magnetic field omitting the Peierls phase [31], since we are not interested in the effect of quantum oscillations itself here. The Hamiltonian reads

$$
\begin{aligned}
& H_{t-J}=-\sum_{i, j, \sigma} t_{i, j} c_{i, \sigma}^{\dagger} c_{j, \sigma}+\frac{J}{2} \sum_{<i, j>}\left(\mathbf{S}_{i} \mathbf{S}_{j}-\frac{n_{i} n_{j}}{4}\right) \\
& -h \sum_{i} S_{i}^{z}
\end{aligned}
$$

where $c_{j, \sigma}$ and $c_{i, \sigma}^{\dagger}$ are the annihilation and creation operators (obeying quasi Fermi statistics [32] due to the doublon prohibition by strong electron correlations) of particle (electron or hole with respect to the chosen representation) with spin $\sigma$ on the site $i, n_{i}=\sum_{\sigma} c_{i \sigma}^{\dagger} c_{i \sigma}$ is the particle number operator, $t_{i, j}$ is the hopping integral, $J$ is the nearest-neighbor exchange integral related with the on-site Coulomb repulsion in the Hubbard model as $J=4 \frac{t^{2}}{U}, \mathbf{S}_{i}$ is the spin operator, $h$ is the energy of a magnetic field.

CPT is a hybrid technique, which provides an effective way to obtain the spectral function within the models for strongly-correlated systems. Within CPT, the first step is to cover the lattice by translations of a cluster. Thus, the full Hamiltonian is represented as $H=H_{c}+H_{c c}$, where $H_{c}$ and $H_{c c}$ are the intracluster and intercluster parts. $H_{c}$ is treated by means of exact diagonalizaion to obtain the cluster Green function. Intercluster interactions are considered then within the Hubbard-I approximation to obtain the site-dependent lattice Green function. Finally, one artificially restores the translational invariance of the electron Green function by transiting to the original Brillouin zone. In such formulation CPT was proposed for the Hubbard model $[27,33]$. In comparison to Quantum Monte Carlo [34] Hubbard-I approximation is qualitatively expected to work in the regime of strong electron correlations $t \ll U$. Since the $t-J$ model is a low-energy effective model for the Hubbard model with parameter $J \sim \frac{t^{2}}{U}$, the approximation should be applicable for the $t-J$ model at $J \ll t$.

Here, we apply a modification of the theory called norm-conserving CPT (NC-CPT), which allows us to keep control over the total quasiparticle weight during the calculation $[19,35]$, covering the lattice with translations of a $2 \times 2$ square cluster. We also introduce the mean fields to consider the canted spin structure, in the same manner as it was done in the papers $[36,37]$ for the Heisenberg model. In the presence of a constant transverse magnetic field applied along the $z$-axis there are two components of magnetization, namely, an in-plane staggered part $\sigma_{x}$ and a uniform part along the field $\sigma_{z}$. The inclusion of mean fields is consistent with the general logic of generalizing CPT in the case of long-ranged order and is needed to break the symmetry of local part $H_{c}$. Particularly, such procedure was shown to produce a correct spin-wave spectrum for the 2D Heisenberg antiferromagnet [36].

Finally, let us introduce the parameters of the $t-J$ model used to obtain the results presented below. We will use the values of hopping integrals $t, t^{\prime}$ and $t^{\prime \prime}$ between the sites of the first, the second and the third coordinate spheres similar to the obtained by fitting the tight binding dispersion curves to the Fermi surfaces of ARPES on LSCO compound: $t \sim 0.25 \mathrm{eV}, t^{\prime} \sim-0.15 t$ and $t^{\prime \prime} \sim-0.5 t^{\prime}$ [12]. We measure the energy in units of $t$ implying $t=0.25 \mathrm{eV}$. We fix $J=0.333 t(U=12 t$ in the Hubbard model), so it corresponds to the typical values $J \sim 0.1 \mathrm{eV}$ for hole-doped cuprates [38]. This value does not seem to be very small compared to the hopping integral. Nevertheless, the comparison of the spectral weight distribution in the Hubbard and $t-J$ models points at qualitative applicability of the $t-J$ model taken with this value of the exchange parameter [39]. Another important parameter is hole doping $p$. It should be pointed out that in the $t-J$ model at fixed $J$ and zero field an increase in $p$ reduces the Neel temperature, so it goes to zero at some value $p_{c}$ [40]. For the relevant parameters $p_{c}$ is similar to the values observed in cuprates, where $p_{c} \sim 0.03$ [38]. Performing calculations at zero temperature, we simply fix a small value of doping $p<p_{c}$, assuming long-range order. 


\section{RESULTS IN NEAREST-NEIGHBOR APPROXIMATION}

First, we discuss the nearest-neighbor case to reveal the main features. In what follows, the amount of doping is $p=0.02$. To obtain the figures presented below, the delta-function was approximated by a Lorentzian with a half-width $\delta$ for the purpose of presenting our results in an ARPES-like manner with finite resolution effects. This parameter is chosen to reproduce the experimental ARPES linewidth.

Fig.1 demonstrates the spectral weight (SW) distribution in the low Hubbard band for different values of a magnetic field, the expectation values of spin projections with respect to the cluster Hamiltonian are also shown. The corresponding density of states (DOS) is presented in Fig.2. It is illustrative to consider the modification of the electronic structure starting from the ferromagnetic case. At $h=4 J$, for the spin-up component we observe the dispersion law specific to a spatially-homogeneous phase. SW is uniform along the dispersion curve. The bandwidth is $8 t$, there is one Van-Hove singularity in the DOS. For spin-down projection there is a narrow band with low SW and without SW at the Fermi level, except the effect of artificial broadening. Decreasing a magnetic field down to $h=3 J$, for example, we observe how the spin-up dispersion is modified by the admixture of different spin states, which causes the redistribution of spectral weight with several dips in the high-energy DOS and decreases the bandwidth. The shape of a spin-down dispersion curve is pretty similar to the spin-up one, but inverted with respect to the $\omega$-axis. It looks like a shadow band in the two-sublattice system. We should emphasize the decreasing SW scale for spindown component with increasing magnetic field. Decreasing a magnetic field further down to $h=0.5 \mathrm{~J}$ when magnetic moments form a slightly tilted AFM structure, at low energy for spin-up component we can recognize the picture similar to a dispersion strongly affected by spin fluctuations as was obtained within different methods $[18,41-46]$. The whole band can be considered as split into two major subbands in agreement with quantum Monte-Carlo calculations [34]. The distributions of SW and DOS for different $z$-projections of spin at this value of field are similar.

Fig. 3 shows the field-induced reconstruction of the FS with spectral line broadening and SW averaging over the energy window similar to ARPES. We see no qualitative changes when varying a magnetic field from zero to $h \approx 0.56 J$. Between $h=0.56 J$ and $h=0.57 J$, as it is evident from Fig.3, the FS undergoes a sharp modification for both spin projections due to a change of the ground state of a cluster in the Hilbert subspace with 3 particles with a jump in magnetization. We emphasize that such small variations of a magnetic field lead to negligible changes in the low-energy electronic structure, except this case. Increasing a magnetic field further up to $h \sim 3 J$, we see a gradual formation of a well-defined hole pocket around $(\pi, \pi)$ with a uniform SW distribution along its arc for spin-up component. For spin-down at the same fields one can see a gradual redistribution of SW at the Fermi level towards the similar hole pocket around $(0,0)$, but with small SW. Fields from $h \approx 3 J$ to $h \approx 4 J$ give no qualitative changes for spin-up component at the Fermi level. Spin-down SW at the Fermi level disappears in the vicinity of saturation.

\section{RESULTS IN CASE OF NON-NEAREST HOPPING}

Let us discuss the case of more realistic model parameters for cuprates. In our calculations with $2 \times 2$ cluster it is possible to account for second-neighbor hopping processes by means of exact diagonalization. Third neighbors are also taken into account, but in terms of perturbation theory. In the previous section we observed the most crucial change of the low-energy structure happening when a cluster's subspace with 3 particles changed its ground state at critical field $h_{c}$. It appears that in case of hole doping an increase in secondneighbor hopping integral $t^{\prime}$ leads to a decrease in $h_{c}$ so that at $t^{\prime} \approx-0.16 t$ critical field goes to zero. Thus, it is possible to observe significant field-induced modification of the FS at fields corresponding to experimentally achievable ones, as presented, for example, in Fig.4, where more realistic hopping parameters are used. For $h=0.02 J$ there is a pseudogap-like picture with a dip of SW in the antinodal direction for both spin-up and spin-down components. The picture is almost the same as in zero field. When a magnetic field is increased by $0.01 J$, the angular SW distribution for spin-up component becomes almost uniform at $h=0.03 J$, while spindown one transforms to a more pronounced pseudogap form. Here, we observe the sharp changes in dispersion as the consequence of an exact account for short-range correlations within a cluster. It might be possible to detect the signatures of such sharp changes in transport measurements on lightly hole-doped cuprates.

\section{CONCLUSIONS}

To conclude, we presented our calculations concerning the field-induced evolution of the electronic struc- 


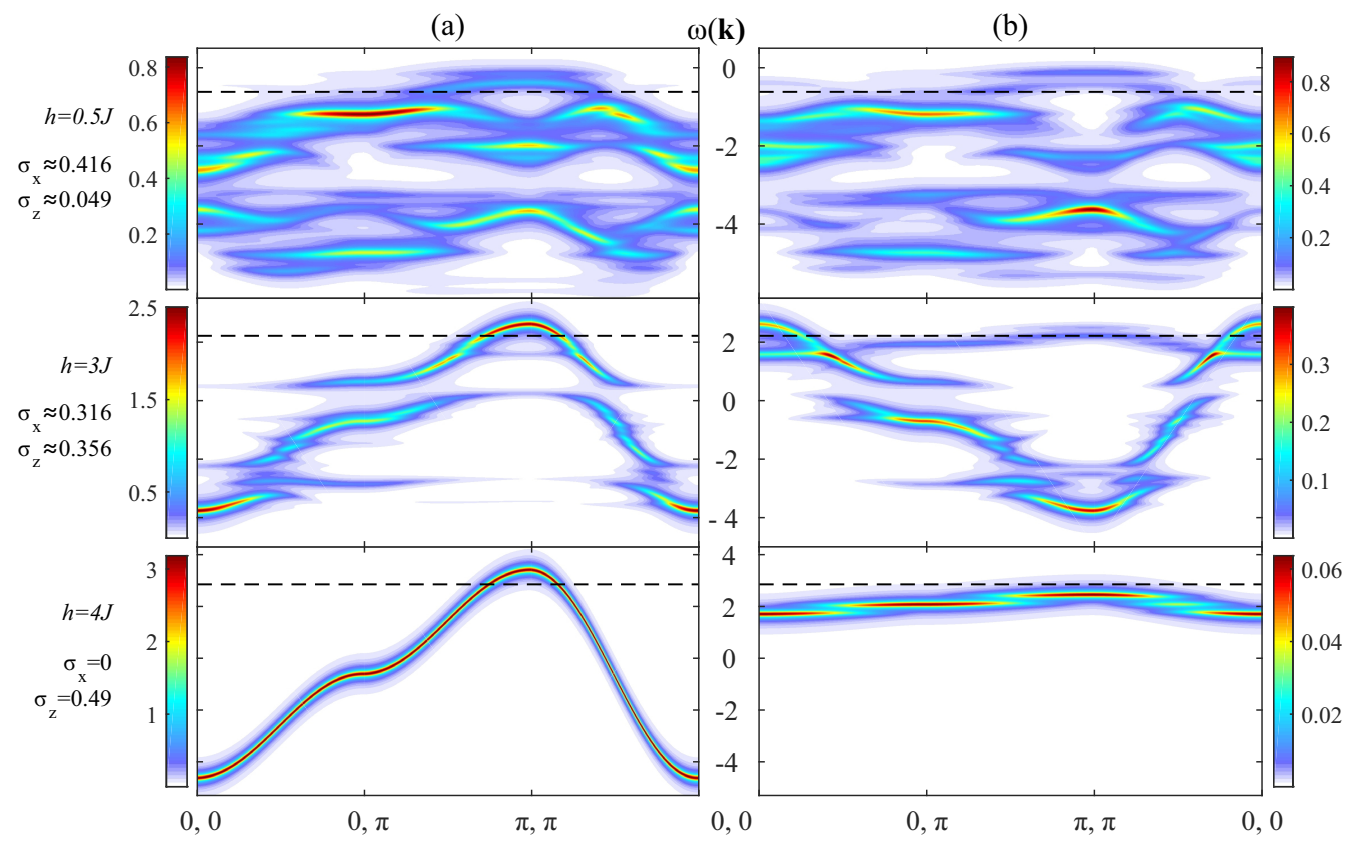

Fig.1.

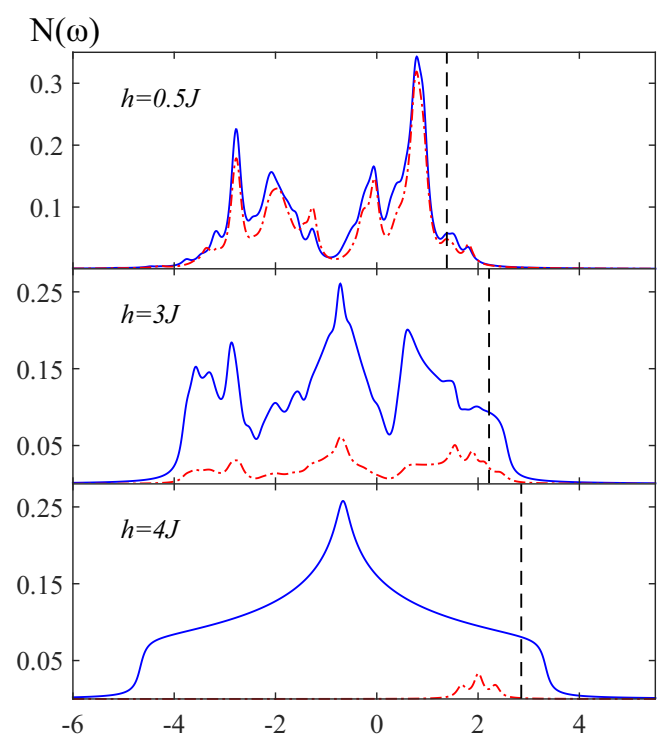

Fig.2.

ture within the $t-J$ model. We have found the nonmonotonic changes of the electronic structure (band dispersion, density of states, and Fermi surface) under increasing magnetic field. From a general point of view, it results from the intrinsic for strongly correlated electrons relation between the electronic and magnetic (a)

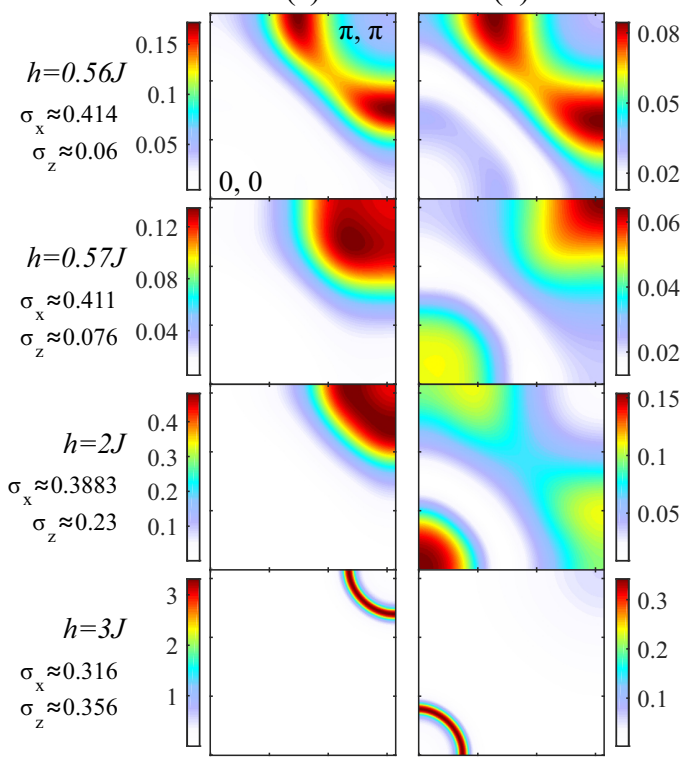

Fig.3.

structures. When spins of nearest atoms are parallel, the interatomic hopping occurs without spin flip similar to free electrons. When nearest spins are antiparallel, the electron hopping requires the spin flip that decreases 


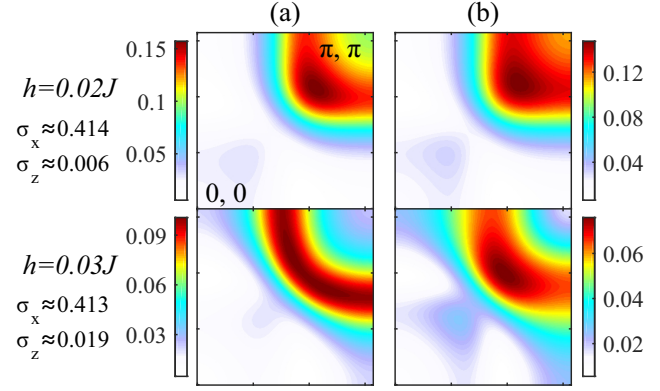

Fig.4.

the hopping probability, decreases the bandwidth, and in some cases may prohibit the interatomic hopping. Nevertheless, before this work it was not shown in details how the electronic and magnetic structure may change in the external magnetic field. We have obtained two main conclusions: i) with increasing magnetic field the sharp change of magnetization and electronic structure occurs, ii) the critical value of magnetic field strongly depends on the fine details of the electronic structure. Thus, in a simplified model with only nearest neighbors hopping the critical filed is unrealistic, $h_{c} \sim 0.5 J \sim 500 \mathrm{~T}$. Nevertheless, in a realistic for cuprates case with non-nearest neighbors hoppings, the critical value appears to be much smaller, here the case when $h_{c} \sim 0.03 J \sim 30 \mathrm{~T}$ was shown for example. As the quantum oscillations have been measured in cuprates in the external fields up to $70 T$, the electronic structure in such large fields and in the absence of the field (when ARPES is measured) may be different and separated by sharp changes that we have found. It is desired to confirm our results in calculations with larger clusters, so that more short-range correlations would be treated exactly.

This work is supported by RSF (Grant No. 14-1200061).

1. N. Doiron-Leyraud, C. Proust, D. LeBoeuf and et al., Nature 447, 565 (2007).

2. D. LeBoeuf, N. Doiron-Leyraud, J. Levallois and et al., Nature 450, 533 (2007).

3. C. Jaudet, D. Vignolles, A. Audouard and et al., Phys. Rev. Lett. 100, 187005 (2008).
4. S. E. Sebastian, N. Harrison, E. Palm and et al., Nature 454, 200 (2008).

5. B. Vignolle, A. Carrington, R. A. Cooper and et al., Nature 455, 952 (2008).

6. C. Jaudet, D. Vignolles, A. Audouard and et al., Phys. Rev. B 82, 140501(R) (2010).

7. P. M. C. Rourke, A.F. Bangura, T. M. Benseman and et al., New J. Phys. 12105009 (2010).

8. T. Helm, M. V. Kartsovnik, M. Bartkowiak and et al., Phys. Rev. Lett. 103, 157002 (2009).

9. N. Barišic̀, S. Badoux, M. K. Chan and et al., Nat. Phys. 9, 761 (2013).

10. A. Sherman, cond-mat/1503.04934.

11. A. Damacelli, Z. Hussein and Z.X. Shen, Rev. Mod. Phys. 75, 473 (2003).

12. T. Yoshida, X. J. Zhou, K. Tanaka and et al., Phys. Rev. B 74, 224510 (2006).

13. T. Yoshida, M. Hashimoto, S. Ideta and et al., Phys. Rev. Lett. 103, 037004 (2009).

14. S. Sakai, Y. Motome and M. Imada, Phys. Rev. Lett. 102, 056404 (2009).

15. S. Sakai, Y. Motome and M. Imada, Phys. Rev. B 82, 134505 (2010).

16. T. D. Stanescu and G. Kotliar. Phys. Rev. B 74, 125110 (2006).

17. M. Civelli, Phys. Rev. B 79, 195113 (2009).

18. M.M. Korshunov and S.G. Ovchinnikov, Eur. Phys. J. B 57, 271 (2007).

19. S. V. Nikolaev, S. G. Ovchinnikov, Zh. Exp. Teor. Fiz. 141, 135 (2012) [J. Exp. Theor. Phys. 114, 118 (2012)].

20. D. LeBoeuf, N. Doiron - Leyraud, B. Vignolle and et al., Phys. Rev. B 83, 054506 (2011).

21. N. Doiron-Leyraud, S. Badoux, S. René de Cotret and et al., Nat. Commun. 6, 6034 (2015).

22. N. Harrison and S. E. Sebastian, Phys. Rev. Lett. 106, 226402 (2011).

23. A. Allais, D. Chowdhury and Subir Sachdev, Nat. Commun. 5, 5771 (2014).

24. T. Wu, H. Mayaffre, S. Krämer and et al., Nature 477, 191 (2011).

25. J. M. Tranquada, B. J. Sternlieb, J. D. Axe and et al., Nature 375, 561 (1995).

26. B. Keimer, N. Belk, R. J. Birgeneau and et al., Phys. Rev. B 46, 14034 (1992).

27. D. Senechal, D. Perez, and M. Pioro - Ladriere, Phys. Rev. Lett. 84, 522 (2000).

28. L. N. Bulaevskii, E. L. Nagaev, D. L. Khomskii, Zh. Exp. Teor. Fiz. 27, 836 (1968). [J. Exp. Theor. Phys. 54, 1562 (1968)]

29. K. A. Chao, J. Spalek, and A. M. Oles, J. Phys. C: Condens. Matter 10, L271 (1977).

30. A. Lüscher and A. M. Läuchli, Phys. Rev. B 79, 195102 (2009). 
31. R. Peierls, Z. Phys. 80, 763 (1933).

32. J. Spalek, Acta Phys. Pol. A 111, 409 (2007).

33. D. Senechal, D. Perez, and D. Plouffe, Phys. Rev. B 66, 075129 (2002).

34. C. Gröber, R. Eder and W. Hanke, Phys. Rev. B 62 4336 (2000).

35. S. V. Nikolaev, S. G. Ovchinnikov, Zh. Exp. Teor. Fiz. 138, 717 (2010) [J. Exp. Theor. Phys. 111, 634 (2010)].

36. V.V. Val'kov and V.A. Mitskan, and G.A. Petrakovskii, Zh. Exp. Teor. Fiz. 129, 268 (2006) [J. Exp. Theor. Phys. 102, 234 (2006)].

37. V. V. Val'kov and V.A. Mitskan, Zh. Exp. Teor. Fiz. 132, 103 (2007) [J. Exp. Theor. Phys. 105, 90 (2007)].

38. A. P. Kampf, Phys. Rep. 249, 219 (1994).

39. V.I. Kuz'min, S. V. Nikolaev and S. G. Ovchinnikov, Phys. Rev. B 90, 245104 (2014).

40. J. L. Richard and V. Yu. Yushankhai, Phys. Rev. B 50, 12927 (1994).

41. A.F. Barabanov, A. A. Kovalev, O. V. Urazaev and et al., Zh. Exp. Teor. Fiz. 119, 777 (2001) [J. Exp. Theor. Phys. 92, 677 (2001)].

42. V. V. Val'kov, D. M. Dzebisashvili, Zh. Exp. Teor. Fiz. 127, 686 (2005) [J. Exp. Theor. Phys. 100, 608 (2005)].

43. M. Aichhorn, E. Arrigoni, M. Potthoff and W. Hanke, Phys. Rev. B 74, 024508 (2006).

44. N. M. Plakida, V.S. Oudovenko, Zh. Exp. Teor. Fiz. 131, 259 (2007) [J. Exp. Theor. Phys. 104, 230 (2007)].

45. A. Avella and F. Mancini, Phys. Rev. B 75, 134518 (2007).

46. A. Avella, Adv. Cond. Mat. Phys. 2014, 515698 (2014).

\section{Figure captions}

Fig.1. (Color online) Electronic spectral function along the symmetric directions of the Brillouin zone in the low Hubbard band within the $t$ - $J$ model for different values of a magnetic field $h$ for (a) spin-up and (b) spin-down components. The spectral lines are approximated by the Lorentzian function with broadening $\delta=0.1 t$. Energy is measured in units of hopping integral $t$. Color-bars represent the correspondence of the colors to the values of spectral function. Here and below the dashed line denotes the position of the Fermi level and we fix the value of the exchange integral $J=0.333$.

Fig.2. (Color online) Density of states for the same parameters as in Fig.1. Blue solid and red dashdotted lines hold for spin-up and spin-down components respectively.

Fig.3. (Color online) Electronic spectral function at the Fermi level in the first quadrant of the Brillouin zone for different values of a magnetic field $h$ for (a) spin-up and (b) spin-down components. The Lorentzian broadening $\delta=0.04 t$ is used. The spectral weight is integrated over the energy window $[-1.5 \delta, 1.5 \delta]$.

Fig.4. (Color online) The same as in Fig.3., but with second-neighbor hopping $t^{\prime}=-0.15 t$ and thirdneighbor hopping $t^{\prime \prime}=0.1 t$. 